\title{
Resistance Expression in Self-pollinated Progenies of Tomato Regenerants Derived from Fusaric Acid-resistant Calli against Fungal Wilt Pathogen Fusarium oxysporum
}

\section{f. sp. lycopersici}

\author{
Kazuyuki Chatani***, Hideyoshi ToyodA*, Yoshinori MatsudA**, \\ Kunihiko SHIMIZU ${ }^{\dagger}$ and Seiji OuCHI*
}

\begin{abstract}
A fungal wilt is a plant disease caused by phytopathogenic filamentous fungi, Fusarium oxyspor$u m$, which invaded the vessel systems of host plants and has been a serious agricultural problem in various major crop plants including tomato. It has been known that various species of Fusarium produced some phytotoxins in infected plants to expand disease symptoms. Fusaric acid(5- $n^{-}$ butylpicolinic acid) (FA) is one of phytotoxins produced by several Fusarium species containing $F$. oxysporum and causes necrotic spots on the leaf blade, shrivelling and drying of leaves, and shrinking and wilting of stem and petioles in tomato cuttings ${ }^{1)}$. In order to protect host plants from Fusarium pathogens, we have attempted an isolation and cloning of FA-detoxyfying genes ${ }^{2,3}$ or application of FA-detoxifying bacteria as a biocontrol agent ${ }^{4}$. In addition, an alternative approach for disease control has been tried to produce the FA-resistant host plants. Shahin and Spivey $^{5}$ reported that tomato plants regenerated from FA-resistant protoplasts exhibited the dominant resistance against $F$. oxysporum $\mathrm{f}$. sp. lycopersici. In order to clarify the mechanisms for disease resistance of host plants, however, the stable expression of the disease resistance acquired should be carefully evaluated during all stages of cultivation, because the invading pathogen can localize latently within host vessel systems for a considerably longer period. In our laboratory, we have succeeded in obtaining FA-resistant tomato callus and intact regenerants derived from FAresistant callus ${ }^{6}$. This progress enables us to examine whether the FA-resistance is effective for exhibiting disease resistance during the whole period of cultivation(for approximately 3 months). The present study describes an expression of the resistance in tomato regenerated from FAresistant calli and their self-pollinated progenies by the field inoculation test with $F$. oxysporum $\mathrm{f}$.
\end{abstract} sp. lycopersici, the causal pathogen of fungal wilt of tomato.

The cultivar "Ponderosa" of tomato (Lycopersicon esculentum Mill, susceptible the pathogen) was purchased from Takii Seed Co., Ltd., Kyoto, Japan, and used in the present study. An induction of friable callus tissues from leaf explants and selection of FA-resistant shoots were conducted according to the method described previously ${ }^{6}$. Namely, surface-sterilized explants were cultured with Murashige-Skoog(MS) medium ${ }^{7)}$ supplemented with $0.01 \mu \mathrm{g} / \mathrm{m} l$ 3-indoleacetic acid(IAA) and $0.1 \mu \mathrm{g} / \mathrm{m} l$ 6-benzylaminopurin (BAP) ( $\mathrm{pH}$ 5.8). Callus clumps were excised and subcultured for several passages at a two-week interval. Callus tissues producing small green spots were cut into small callus clumps and transferred to the shoot differentiation medium (MS medium with $0.1 \mu \mathrm{g} /$ $\mathrm{m} l$ IAA and $1.0 \mu \mathrm{g} / \mathrm{m} l$ BAP) containing $50 \mu \mathrm{g} / \mathrm{m} l$ FA (Sigma Chemical Co., St. Louis, MO, USA) .

\footnotetext{
* Faculty of Agriculture, Kinki University, 3327-204 Nakamachi, Nara 631, Japan

** Institute for Comprehensive Agricultural Science, Kinki University, 3327-204 Nakamachi, Nara 631, Japan

*** Present address: Fuji-Oyama Research Laboratory, Dai-ichi Engei Plantech, Shizuoka 410-13, Japan

+ Present address: Chonan-Breeding Farm, Kyowa Seed, Chosei-gun, Chiba 297-01, Japan
} 
Table 1. Segregation of fusaric acid(FA) resistance in self-pollinated progenies of fusaric acid-resistant $\mathrm{R}_{0}$ lines.

\begin{tabular}{cccc}
\hline \multirow{2}{*}{$\begin{array}{l}\text { Selfed FA-resistant } \\
\mathrm{R}_{0} \text { lines }\end{array}$} & \multicolumn{2}{c}{$\begin{array}{l}\text { Number of FA-treated } \mathrm{R}_{\mathbf{1}} \\
\text { progenies }\end{array}$} & $\begin{array}{l}\text { P value }(3: 1) \\
\text { between }\end{array}$ \\
\cline { 2 - 3 } & Resistant & Susceptible & \\
\hline $\mathrm{R}_{0}-01$ & 38 & 15 & $0.50-0.75$ \\
$\mathrm{R}_{0}-12$ & 55 & 17 & $0.75-0.90$ \\
$\mathrm{R}_{0}-22$ & 69 & 24 & $0.75-0.90$ \\
$\mathrm{R}_{0}-40$ & 37 & 14 & $0.50-0.75$ \\
$\mathrm{R}_{0}-88$ & 54 & 21 & $0.50-0.75$ \\
\hline
\end{tabular}

FA-resistant shoots were transferred to hormone-free MS medium for root initiation, and regenerated plants were transplanted to soil and acclimated in a moistened chamber for a week. Resistance of regenerated plants and their self-pollinated progenies to FA was examined by dipping excised upper branches possessing five leaves into the aqueous solution containing $300 \mu \mathrm{g} / \mathrm{m} l$ of $\mathrm{FA}^{7)}$.

In the present study, we finally obtained five regenerated plants $\left(R_{0}\right.$ generation) resistant to FA and self-pollinated these regenerants to obtain FA-resistant $R_{1}$ progenies. Table 1 reveals the segregation of resistance and susceptibility to $F A(300 \mu \mathrm{g} / \mathrm{m} l)$ in $R_{1}$ progenies. The data indicate that these lines had monogenic segregation of 3 resistant $: 1$ susceptible by self pollination. This result suggests that the FA resistance is probably due to a dominant single gene mutation, and this mutation was heterozygously induced in the present $R_{0}$ lines. In order to obtain homozygous and heterozygous resistant plants, we further self-pollinated these FA-resistant $\mathrm{R}_{1}$ plants and selected the homozygous (showing the FA resistance in all of progenies) and heterozygous lines (containing both FA-resistant and FA-susceptible plants). A similar procedure for self-pollination was repeated, and finally the homozygous $\left(R_{2} F R-1208,-4013,-8835\right)$ and heterozygous $R_{2}$ plants $\left(R_{2} F R-\right.$ 0232) were obtained. Using these tomato plants, we examined their resistance expression to the fungal pathogen inoculated. Inoculation of the pathogen was conducted by the method reported previously ${ }^{8}$. Thirty-day-old tomato plants (25 plants of each line) were removed from the soil, and the roots were dipped for 3-5 min in a slurry of $F$. oxysporum f. sp. lycoperisci, Race I(density of microconidia, $3-5 \times 10^{6}$ spores $/ \mathrm{m} l$ ). The inoculated plants were planted in moist soil and grown in a green house controlled at $28-30^{\circ} \mathrm{C}$. The appearance of wilt disease was scored by recording the occurrence of some disease symptoms (vein necrosis, yellowing of lower leaves, and partial and complete wilting), 1 and 3 months after inoculation with the pathogen. As shown in Table 2, control plants (Fusarium-susceptible parental line and FA-susceptible regenerants) infected by the pathogen showed vein necrosis and leaf yellowing within 15-20 days and completely wilted within 1 month after inoculation. These results suggest that the present inoculation was effective for infecting test plants with the pathogen without escape and therefore for evaluating the expression of disease resistance in the present FA-resistant plants. The present data clearly indicated that both homozygous and heterozygous FA-resistant plants were strongly resistant to the inoculated pathogen at the earlier stage(1 month after inoculation), though a few plants showed a slight yellowing of the lower leaves. Shahin and Spivey ${ }^{5}$ examined the disease resistance of tomato plants regenerated from FA-resistant protoplasts and their self-pollinated $R_{1}$ and $R_{2}$ progenies, at the similar stage after inoculation (approximately 1 month after inoculation) and described a single dominant gene for Fusarium-wilt resistance in these plants. These results were coincident with the data obtained in the present work. Nevertheless, the present study indicated that the gene for 
Table 2. Inoculation of fusaric acid-resistant $\mathrm{R}_{2}$ lines with $F$. oxysporum $\mathrm{f}$. sp. lycopersici, Race I*1.

\begin{tabular}{|c|c|c|c|c|}
\hline \multirow{3}{*}{ Plants used } & \multicolumn{4}{|c|}{ Number of plants } \\
\hline & \multicolumn{2}{|c|}{1} & \multicolumn{2}{|c|}{$3^{* 2}$} \\
\hline & Resistant & Susceptible & Resistant & Susceptible \\
\hline \multicolumn{5}{|l|}{$\mathrm{R}_{2}$ lines } \\
\hline $\mathrm{R}_{2}$ FR-1208 & $24\left(2^{* 3}\right)$ & 1 & 2 & 23 \\
\hline $\mathrm{R}_{2} \mathrm{FR}-4013$ & $18(1)$ & 7 & 0 & 25 \\
\hline $\mathrm{R}_{2} \mathrm{FR}-8835$ & $23(1)$ & 2 & 0 & 25 \\
\hline $\mathrm{R}_{2} \mathrm{FR}-0232^{* 4}$ & $22(1)$ & 3 & 0 & 25 \\
\hline \multicolumn{5}{|l|}{ Control } \\
\hline Parental line & 0 & 25 & - & - \\
\hline $\mathrm{R}_{1} \mathrm{FS}-0011^{* 5}$ & 0 & 25 & - & - \\
\hline
\end{tabular}

${ }^{* 1}$ Inoculated plants showing disease symptoms described in the text were regarded as susceptible plants and, asymptomatic, healthy plants as resistant ones to the disease.

*2 Months after inoculation.

${ }^{* 3}$ Number of plants showing slight leaf yellowing.

${ }^{* 4}$ Heterozygous FA-resistant line.

*5 FA-susceptible $\mathrm{R}_{1}$ line.

FA-resistant plants did not express the resistance to the pathogen at the later stage when tomato fruits were harvested (3 months after inoculation) (Table 2). Namely, both the homozygous and heterozygous FA-resistant plants showed the yellowing of lower leaves and vein necrosis or partial wilting at approximately 2 months and then completely wilted 3 months after inoculation. These results suggest that the fungal wilt could be induced by multiple phytopathogenic attacks of the pathogen including the production of FA at the earlier stage, and therefore tomato plants would be required to acquire resistance against these subsequent fungal attacks in order to effectively protect tomato plants from the disease during the entire period of cultivation.

(Accepted November 18, 1995)

\section{References}

1) Kern, H., 1972. In "Phytotoxins in Plant Diseases" (eds. by Wood, S., et al.), p. 35-48, Academic Press, New York.

2) Utsumi, R., T. Hadama, M. Noda, H. Toyoda, H. Hashimoto, S. Ouchi, 1988. J. Biotechnology, 8: 311-316.

3) Toyoda, H., K. Katsuragi, T. Tamai, S. Ouchi, 1991. J. Phytopathol., 133: 265-277.

4) Ouchi, S., H. Toyoda, R. Utsumi, H. Hashimoto, T. Hadama, 1989, In "Phytotoxins and Plant Pathogenesis" (eds. by Graniti, A., et al.), p. 301-317, Springer-Verlag, New York.

5) Shahin, E. A., R. Spivey, 1986. Theor. Appl. Genet., 73: 164-169.

6) Toyoda, H., Y. Matsuda, K. Shimizu, H. Ogata, H. Hashimoto, S. Ouchi, 1988. Plant Tissue Culture Lett., 5: $66-71$.

7) Murashige, T., F. Skoog, 1962. Physiol. Plant., 15: 473-497.

8) Toyoda, H., H. Hashimoto, R. Utsusmi, H. Kobayashi, S. Ouchi, 1988. Phytopathology, 78: 1307-1311. 